

1) Department of Conservative Dentistry, University of Medicine and Pharmacy, Cluj-Napoca, Romania

2) Department of Oral Rehabilitation, Iuliu Hatieganu University of Medicine and Pharmacy, ClujNapoca, Romania

3) Department of Restorative Dentistry, Marmara University, Istanbul, Turkey

\section{The advantages of the dental operative microscope in restorative dentistry}

Marius Bud ${ }^{1}$, Stefan Jitaru ${ }^{1}$, Ondine Lucaciu² ${ }^{2}$, Bora Korkut ${ }^{3}$, Lucia Dumitrascu-Timis $^{1}$, Corina Ionescu ${ }^{1}$, Sanda Cimpean ${ }^{1}$, Ada Delean ${ }^{1}$

\begin{abstract}
Magnification devices improve direct and indirect vision and precision being significantly higher in microscope use compared to the loupes. Dental loupes are the most commonly used devices for magnification, due to the more affordable prices and the ease of use without major changes in the working protocol and ergonomics. Loupes primary benefits reported refers to ergonomics and posture, restoration evaluation/detection and overall treatment quality. There are some disadvantages that limit the use of loupes among dentists: lack of fixed position (fine movements of the dentist's head disturb the image of the magnified operating field); the need to change the loupes to achieve different magnification. On the contrary, the use of a dental microscope require minimum adjustment and effort so as to reduce postural deviation while working.

It has been more than 30 years since dental practitioners raised the issue of using dental operating microscopes (DOM) in restorative dentistry, identifying the benefits of magnification in diagnosis and treatment steps. The growth is evident in recent years, maybe because of the familiarity of dentists with dental microscopes which have already become implicit endowment for endodontic specialists. Fiber optic lighting is the key factor that complements the magnification offered by the microscope so that treatments can now be performed under increased safety conditions and in conditions of significantly higher quality than in the past.

Since the beginning of the 2000s the principles of minimally invasive dentistry have been widely promoted in dentistry. These principles are major impetus for the use of microscope by dentists. Working with magnification leads dentists to be more conservative with dental tissues.
\end{abstract}

Keywords: magnification, dental operating microscope, restorative dentistry, microscope restorations

The basic principles of restorative dentistry have not changed significantly in the last 30 years, but aesthetic, biological and mechanical criteria are now more strictly applied by dentists. Moreover, this tendency has been amplified by the quick evolution of the dental materials, equipment and techniques. Nowadays dentists do more aesthetic, biomimetic and predictable treatments that require less chair time with an increased success rate. These are difficult to be achieved without the use of magnification and also a good lighting.
Since the 1970s, dentists have begun to consider the use of magnification in dentistry. Both microscopes and loupes have been widely adopted by dentists. Magnification can be divided in lowmagnification $(2 \mathrm{x}-8 \mathrm{x})$, mid-magnification $(8 \mathrm{x}-16 \mathrm{x})$, and high-magnification (16x25x). Microscopes provide adjustable magnification (magnification range $4 x-25 x$ ), while most loupes provide fixed magnification (magnification range $2.5 \mathrm{x}-6 \mathrm{x})$.

Magnification devices improve direct and indirect vision and precision 
being significantly higher in microscope use compared to the loupes [1]. Dental loupes are the most commonly used devices for magnification, due to the more affordable prices and the ease of use without major changes in the working protocol and ergonomics. They are available in multiple variants and models offering magnifications up to $6.5 \mathrm{X}$, the most used being $2.5 \mathrm{x}$ [2].

The reported primary benefits of loupes refer to ergonomics and posture, restoration evaluation/detection and overall treatment quality [3]. There are some disadvantages that limit the use of loupes by dentists: lack of fixed position (fine movements of the dentist's head disturb the image of the magnified operating field); the need to change the loupes to achieve different magnification. On the contrary, the use of a dental microscope requires minimum adjustment and effort so as to reduce postural deviation while working.

It has been more than 30 years since dental practitioners raised the issue of using dental operating microscopes (DOM) in restorative dentistry, identifying the benefits of magnification in the diagnosis and treatment steps [4]. However, the effective widespread introduction of microscope use in restorative dentistry has evolved quite slowly. The growth is evident in recent years, maybe because of the familiarity of dentists with dental microscopes which have already become common tools for endodontic specialists [5]. Fiber optic lighting is the key factor that complements the magnification offered by the microscope so that treatments can now be performed under increased safety conditions and in conditions of significantly higher quality than in the past.

Since the beginning of the 2000s the principles of minimally invasive dentistry have been widely promoted in dentistry [6]. These principles represent major impetus for the use of microscope by dentists. Working with magnification leads dentists to be more conservative with dental tissues.

David Clark, founder of the Academy of Dental Microscopy and one of the pioneers of dental microscopy, said that "the greatest indicator of long-term retention of teeth is the volume of healthy natural dental tissue that remains after we finish treating a tooth" [7].

There are some mandatory requirements for using a dental microscope:

1. Working in indirect vision using a good dental mirror. The of maxillary and mandibular posterior teeth is impossible in direct view when the dentist is located between the 11:00 to the 12:30 position, for right-handed operators.

2. Using rubber-dam to prevent mirror fogging. Beside offering a good isolation, the rubber-dam keeps soft tissues retracted (tongue, lips) in order to have a wide working field.

3. Team work with a dental assistant to pass the instruments directly into the dentist's hand, as the dentist's eyes are fixed in the eyepiece and no longer able to reach for the instruments while working.

4. An up-mounted dental unit with all hand pieces situated above the patient's chest during treatment, which provides the assistant with access to change burs and rotary instruments and pass the air spray in low and high speed directly to the dentist.

5. Using of a dental chair with armrests. This allows the operator to have an important fixed point in the elbow in order to make fine and small amplitude movements. Chair should have important feature like adjustable height, width, tilt, backrest, seat pan and armrests.

6. Positioning the frequently used items close to the point of use (50-60 $\mathrm{cm}$ for most people) and not above or below waist height [8].

The benefits of DOM use in dentistry are now unanimously accepted:

\section{Increased vision}

Due to the magnification and transmitted light by fiber optics to the operating field, it is possible to treat more precisely because of improved visibility compared to the direct eye view [9].



Figure 1. Eyepieces, Optical body, Light source.

\section{Improve in overall quality of the treatment}

It was proven that dentist do better restorative treatments under microscopic view due to the precision of the diagnosis and the proper completion of the treatment steps [10-14]. A magnified image of the operative field may help the dentist to check cavity preparations, matrix fitting, saliva infiltration, remaining dentine debris, composite layering, occlusal morphology, macro/micro details, surface texture of restoration, air voids, impurities and over-contours in a better way. 


\section{Minimally invasive treatment with less dental hard tissue removal}

The principles of minimally invasive dentistry have now become a priority for almost every dentist. It is easier to be achieved with magnification. Early detection of incipient enamel cavities before their expansion is impossible with the naked eye without magnification and the same is the removal of old filling materials with respect for the remaining healthy dental tissue.

\section{Lesser time for the final occlusion check}

Using the magnification in occlusal layering step, the operator may reduce the over-contoured resin material applied at the level of occlusal surfaces. Therefore that minimizes the occlusal adaptation time.

\section{Ergonomic working position}

Dental practitioners who use dental operating microscope declare the drastic decrease of the eye fatigue, musculoskeletal pains and the decrease of the psychological fatigue. This is because of the magnified image of the operating field, the increase of the lighting intensity, the ergonomic position during the work and the certainty of the control of the procedures performed. All these bring a net benefit of the dentist's quality of life [14-18].

\section{Comfort and motivation of the dentist}

The use of a microscope increases the certainty of approaching certain treatments through the confidence of success brought by the possibility of controlling and verifying the details that are important in each treatment step, from cleaning and preparing the cavities to the finishing and polishing protocols. assistant

7. Better communication with patient and dental

The use of the dental operating microscope offers the possibility to take pictures and video data that can also be useful for the patient's motivation and information about his/her treatment plan. The data are meant to make the patient better and easier understand the explanations and may also be necessary for legal issues. Moreover, in 4-hands work, the nurse is more efficient if she/he can follow directly (either on the screen or through a dedicated eyepiece) the operating field as the dentist sees it. That allows the assistant to intervene promptly at certain stages where the aid is directly necessary (for adapting a matrix, fixing a dam clamp, etc.)

\section{Avoid of iatrogenic damages}

Working in a well-lit and around 20 times magnified operating field, gives the dentist the opportunity to be extremely precise in movements while using aggressive rotary instrumentation, especially burrs at high speeds. Thus, it is possible to avoid damaging the adjacent teeth during proximal cavity preparations, prosthetic crown preparations or to avoid deep dentin layer exposures leading to the opening of the pulp chamber, etc. These situations are, in many cases, difficult or impossible to control without a good magnification and effective lighting, especially when the operating field is visually obstructed by presence of cooling water mixed with enamel and dentin debris.

The benefits of magnification in restorative dentistry treatments are important in the following situations:

- Identification of areas where enamel tissue is demineralized (Figure 2)

- Identification and minimally invasive removal of old restorations (Figure 3)

- Gaps or impurities in restorative materials

- Inspection of caries borders and remaining caries tissues

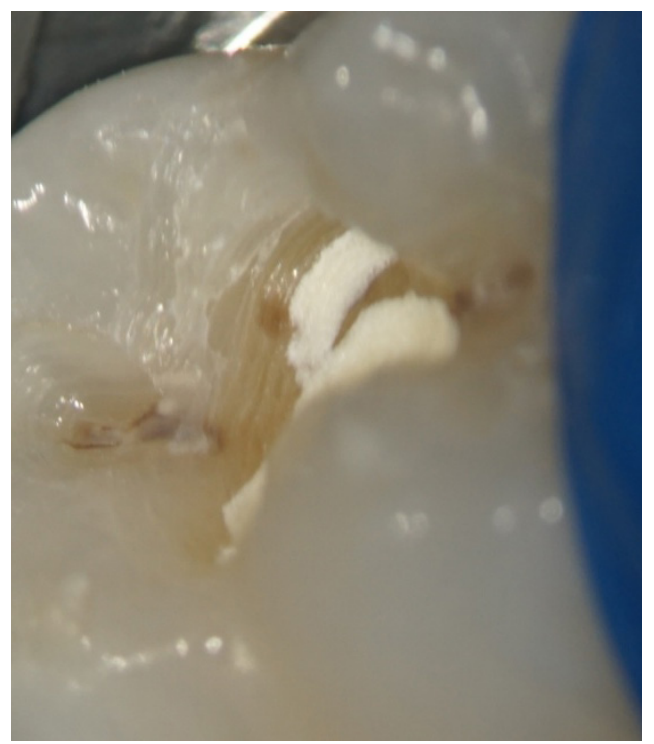

Figure 3. Old materials removal. 


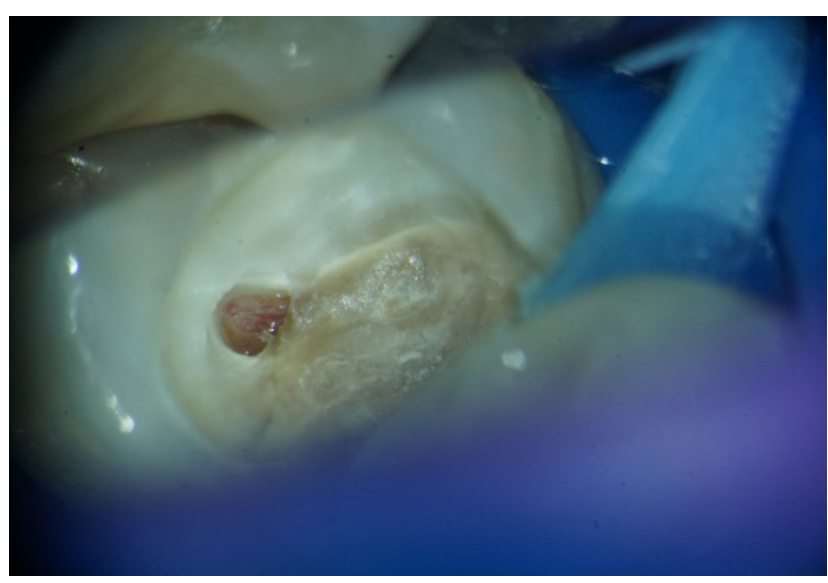

Figure 4. Pulp chamber openning.

- Management of small, accidental pulp chamber openings without mechanical injury of the pulp (Figure 4)

- Identification of enamel cracks or fractures especially located on the gingival margin in Class II and MOD cavities (Figure 5)

- Checking of the sectional matrices adaptation and controlled application of liner (Figure 6)

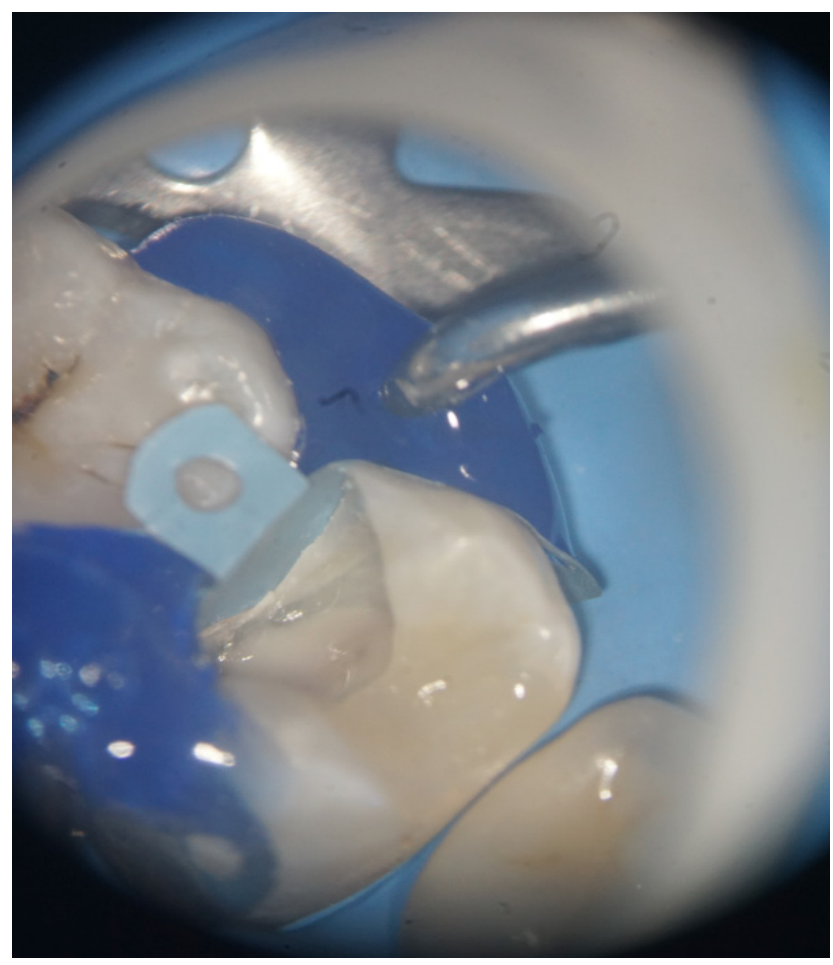

Figure 6. Sectional matrices adaptation.

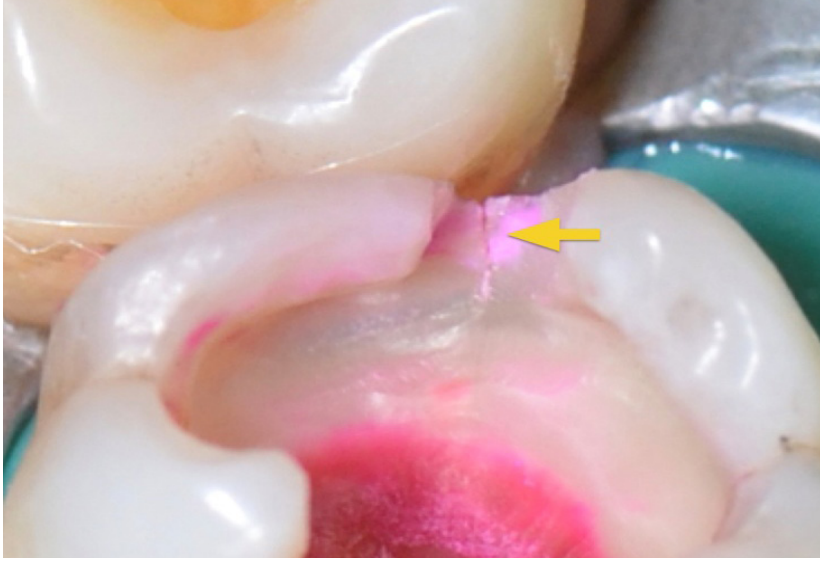

Figure 5. Enamel cracks.

- Evaluation of the marginal gaps of the restorations

- Application of the liner for pulp protection selectively

- Minimally invasive removal of amalgam restorations

- Minimally invasive preparation of small Class III cavities (Figure 7)
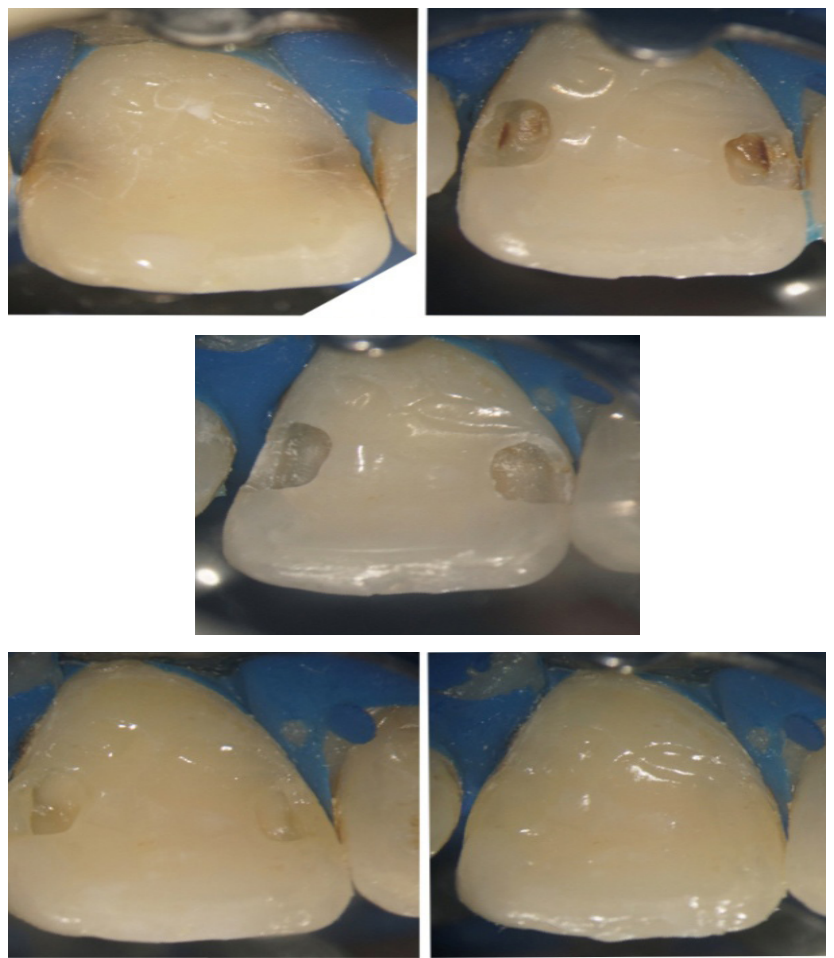

Figure 7. Preparation of small Class III cavities. 
- Evaluation of the preparation edges for inlay / onlay and overlay restorations (Figure 8)

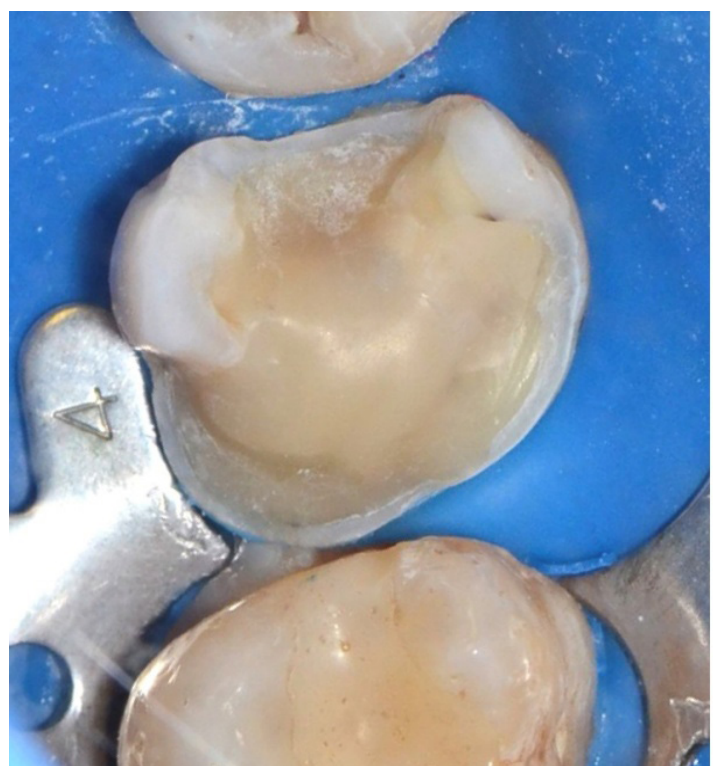

Figure 8. Preparation edges for indirect restoration.

- Removal of excess composite materials

- Adhesive excess removal [19]

- Check if the composite is flowing into all aspects of the preparation, in al irregularities

- Detect microscopic air bubbles in flowable composite, located either inside or on the external surface or margin of the flowable composite bolus [20]

- Good finishing of margins [21]

- Simplifying the finishing and polishing working protocol [22]

- Improve the marginal integrity of proximal composite restorations [23].

There is a variety of additional upgrades for core microscope functions. Variable focal distance adapters have become available instead of fixed focal distances that limit the microscope to an object distance of $20-30 \mathrm{~cm}$. These are often offered in top-of-the-line microscopes. Extendable (foldable) binoculars were introduced for improved ergonomics. A beamsplitter for a second eyepiece may be added in order to allow the assistant or another dentist/student to observe the operating field during the treatment. A beamsplitter for a photo/video camera allows the dentist to take photos and videos for the documentation of cases or for self-education of the dental team or students [24].

There are also a number of factors that limit the widespread use of microscope. The most important is the extremely high cost. However, there may also be problems like office space limitations or low motivation to use the microscope due to the lack of knowledge. Whatever the impediments, microscopy takes an increasing advantage for patients and dentists day by day.

The history of other medical specialties has already showed that new equipment may be hard to implement in daily practice. There is a fine balance between three major factors: the benefits to the treatment, the cost of acquisition and the effort made by the dentist for using it. In the case of microscopes, until now, only the first factor is unquestionable. Costs and reluctance to change will remain to be the major obstacles in spreading the use of microscopes in restorative dentistry for now.

\section{References}

7. Eichenberger M, Biner N, Amato M, Lussi A, Perrin P. Effect of Magnification on the Precision of Tooth Preparation in Dentistry. Oper Dent. 2018;43:501-507.

8. Farook SA, Stokes RJ, Davis AK, Sneddon K, Collyer J. Use of dental loupes among dental trainers and trainees in the UK. J Investig Clin Dent. 2013;4:120-123.

9. Hayes $\mathrm{MJ} \mathrm{BOH}$ BHSc(Hons) MEd $\mathrm{PhD}$, Rogers $\mathrm{AA}$ BOH GDipPsych (Hons), Chuanon J BOH BPharm GradCertPharmPrac, Tan T BOH, Lai I BOH, Yong E BOH. Dental and oral health students' perceptions of loupes. Int J Occup Saf Ergon. 2019:1-26.

10. Jurkschat U. Improvement of stomatological diagnosis by means of the II operating microscope. Stomatol DDR. 1979;29:15-18.

11. Bowers DJ, Glickman GN, Solomon ES, He J. Magnification's effect on endodontic fine motor skills. J Endod. 2010;36:1135-1138.

12. Perrin P, Jacky D, Hotz P. The operating microscope in dental practice: minimally invasive restorations. Schweiz Monatsschr Zahnmed. 2002;112:722-732.

13. Clark D. The new science of strong teeth: Class II Preps, Dentistry Today. June 2014. Available from: https:/www. dentistrytoday.com/restorative-134/9286-the-new-scienceof-strong-teeth-class-ii-preps

14. Jodalli PS, Kurana S, Shameema, Ragher M, Khed J, Prabhu V. Posturedontics: How does dentistry fit you? J Pharm Bioallied Sci. 2015;7(Suppl 2):S393-S397.

15. Branson BG, Bray KK, Gadbury-Amyot C, Holt LA, Keselyak NT, Mitchell TV, et al. Effect of magnification lenses on student operator posture. J Dent Educ. 2004;68:384-389.

16. Baldassari-Cruz LA, Lilly JP, Rivera EM. Effectiveness of mesiolingual canal location with and without the use of the microscope. J Endod. 1998;24:287.

17. Rubinstein RA, Kim S. Short-term observation of the results of endodontic surgery with the use of a surgical operating microscope and Super-EBA as rootend filling material. J Endod 1999;25:43-48.

18. Rubinstein RA, Kim S: Long-term follow-up of cases considered healed one year after apical microsurgery. J 
Endod. 2002;28:378-383.

19. Stropko JJ. Canal morphology of maxillary molars: clinical observations of canal configurations. J Endod. 1999;25:446450 .

20. Hagge MS. Use of surgical telescopes by senior dental students: a survey. J Prosthodont. 2003;12:271-279.

21. Sunell S, Rucker L. Surgical magnification in dental hygiene practice. Int J Dent Hyg. 2004;2:26-35.

22. Chang BJ. Ergonomic benefits of surgical telescope systems: selection guidelines. J Calif Dent Assoc. 2002;30:161-169.

23. Kinomoto Y, Takeshige F, Hayashi M, Ebisu S. Optimal positioning for a dental operating microscope during nonsurgical endodontics. J Endod. 2004;30:860-862.

24. Comes C, Valceanu AS, Rusu D, Didilescu A, Bucur A, Anghel $M$ et al. A Study on the Ergonomical Working Modalities using the Dental Operating Microscope (DOM). Part II: Ergonomic Design Elements of the Operating Microscopes. Timisoara Medical J. 2010;60:97-102
25. van As GA. The use of extreme magnification in fixed prosthodontics. Dent Today. 2003;22:93-99.

26. Mamoun J. Preparing and Restoring Composite Resin Restorations. The Advantage of High Magnification Loupes or the Dental Surgical Operating Microscope. N Y State Dent J. 2015;81:18-23.

27. Sheets CG. The periodontal-restorative interface: enhancement through magnification. Pract Periodontics Aesthet Dent. 1999;11:925-931; quiz 932.

28. Pascotto RC, Benetti AR. The clinical microscope and direct composite veneer. Oper Dent. 2010;35:246-249.

29. Reddy P, Jain V, Kaushik M, Roshni, Mehra N, Rana R, et al. Assessment of Marginal Integrity of Proximal Composite Resin Restorations Performed with or without Magnification. Journal of Clinical and Diagnostic Research. 2017;11:ZC01ZC04.

30. Das UK, Das S. Dental operating microscope in endodontics-a review. IOSRJDMS. 2013;5:1-8. 